LETTER

(Anti-) de Sitter electrically charged black-hole solutions in higher-derivative gravity

To cite this article: Kai Lin et al 2016 EPL 11460006

View the article online for updates and enhancements.
Related content

- Gravitational quasinormal modes for anti-
$\frac{\text { de Sitter black holes }}{\text { lan G Moss and James P Norman }}$
- Gravitational quasinormal modes for Kerr
$\frac{\text { anti-de Sitter black holes }}{\text { M Giammatteo and lan G Moss }}$
- The portrait of eikonal instability in
Lovelock theories
R.A. Konoplya and A. Zhidenko

Recent citations

- Quasinormal modes of the four-
$\frac{\text { dimensional black hole in Einstein-Weyl }}{\text { gravity }}$
A. F. Zinhailo
- Quadratic Gravity
Alberto Salvio
- Quantum loop corrections of charged dS
$\frac{\text { black hole }}{\text { J. Naji }}$




\title{
(Anti-) de Sitter electrically charged black-hole solutions in higher-derivative gravity
}

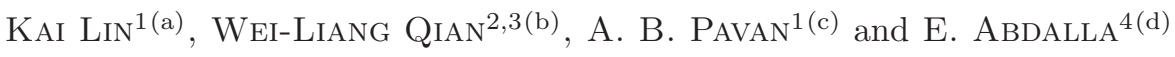 \\ ${ }^{1}$ Instituto de Física e Química, Universidade Federal de Itajubá - Itajubá, MG, Brasil \\ ${ }^{2}$ Escola de Engenharia de Lorena, Universidade de São Paulo - Lorena, SP, Brasil \\ ${ }^{3}$ Faculadade de Engenharia de Guaratinguetá, Universidade Estadual Paulista - Guaratinguetá, SP, Brasil \\ ${ }^{4}$ Instituto de Física, Universidade de São Paulo - CP 66318, 05315-970, São Paulo, SP, Brasil
}

received 4 June 2016; accepted in final form 1 July 2016

published online 15 July 2016

PACS 04.70.Bw - Classical black holes

PACS 04.25.dg - Numerical studies of black holes and black-hole binaries

PACS 04.60.-m - Quantum gravity

\begin{abstract}
In this paper, static electrically charged black-hole solutions with cosmological constant are investigated in an Einstein-Hilbert theory of gravity with additional quadratic curvature terms. Beside the analytic Schwarzschild (Anti-) de Sitter solutions, non-Schwarzschild (Anti-) de Sitter solutions are also obtained numerically by employing the shooting method. The results show that there exist two groups of asymptotically (Anti-) de Sitter spacetimes for both charged and uncharged black holes. In particular, it was found that for uncharged black holes the first group can be reduced to the Schwarzschild (Anti-) de Sitter solution, while the second group is intrinsically different from a Schwarzschild (Anti-) de Sitter solution even when the charge and the cosmological constant become zero.
\end{abstract}

Copyright (C) EPLA, 2016

Though Einstein's general relativity has been extensively tested at the highest achievable experimental precision up to date, gravity is not a renormalizable quantum field theory from the theoretical viewpoint. A possible attempt to solve the problem of the non-renormalizability of general relativity is to include higher-order corrections that become important at higher energy [1]. In this context, general relativity can be viewed as an effective lowenergy field theory, which is understood to be largely independent of the details in higher energy scales. It can be shown when all possible quadratic curvature invariants are added to the Einstein-Hilbert action, a renormalizable theory is achieved at the cost of the presence of the ghost modes [2]. Thus the study of the properties of such higherderivative gravity could shed light on the ongoing efforts to understand the nature of gravity.

In order to better understand such a new theory of gravity, it seems of considerable interest to investigate the behaviour of black-hole solutions, since they are fundamental objects in general relativity. Such studies have also been

\footnotetext{
(a) E-mail: 1k314159@hotmail.com

(b) E-mail: wlqian@usp.br

(c) E-mail: alan@unifei.edu.br

(d) E-mail: eabdalla@usp.br
}

performed in other theories of gravity containing higherorder curvature corrections with well-defined coefficients, such as Lovelock theory [3]. Moreover, the question of stability of charged black holes is relevant and has been intensively studied recently in recent years [4,5].

Recently, Lü et al. found, numerically, a nonSchwarzschild solution in a theory of gravity with a quadratic Weyl scalar on action [6,7], and revealed that this higher-derivative gravity possesses additional static spherically symmetric black-hole solution compared to Einstein's general relativity. We also showed in [8] that electrically charged static solutions in this theory are also characterized by two groups of solutions. These solutions reduce, respectively, to Schwarzschild black hole and nonSchwarzschild black hole solutions, when the charge of the black hole goes to zero. Surprisingly the ReissnerNordström metric is not a solution of the field equations when the coefficients of the higher-derivative terms do not vanish.

Being first introduced by Einstein to describe a static universe and later abandoned by himself after Hubble observes the expansion of the universe, the cosmological constant is a measure of the value of the energy density of the vacuum of space and could be a candidate to explain the 
expansion. In addition, the discovery of the accelerating universe after 1990 from distant supernovae implies that more than half of the energy density of the universe can be attributed to the unkown so-called dark energy. Thus, while poorly understood at a fundamental level, the concept of cosmological constant was revived and is, by far, the simplest possible form of dark energy. Another important motivation in studying spacetimes with cosmological constant, namely negative one, is the AdS/CFT correspondence [9]. Therefore, it seems to be interesting to investigate the properties of the non-Schwarzschild black-hole solutions with the presence of the cosmological constant. In this letter, we carry out a study of the (Anti-) de Sitter electrically charged black-hole solutions in higher-derivative gravity.

The action of higher-derivative gravity in EinsteinHilbert theory with quadratic curvature scalars and with electromagnetic field and the cosmological constant $\Lambda$ can be written as

$$
\mathcal{L}=\gamma R-2 \Lambda-\alpha C_{\mu \nu \rho \sigma} C^{\mu \nu \rho \sigma}+\beta R^{2}-\kappa F_{\mu \nu} F^{\mu \nu},
$$

where $F_{\mu \nu}=\nabla_{\mu} A_{\nu}-\nabla_{\nu} A_{\mu}$ is the electromagnetic tensor, $C_{\mu \nu \rho \sigma}$ is the Weyl tensor, $\alpha, \beta, \gamma$ and $\kappa$ are coupling constants.

According to the analysis in $[6,8,10]$, (non-)charged black-hole solutions without cosmological constant are independent of the $\beta R^{2}$ term since the additional terms on the action are traceless. Here, this condition is broken because of the introduction of the cosmological constant. However, we set $\beta=0$ for the sake of convenience. Thus, the resulting field equations are given by

$$
\begin{aligned}
R_{\mu \nu}-\frac{1}{2} g_{\mu \nu} R-\Lambda g_{\mu \nu}-4 \alpha B_{\mu \nu}-2 \kappa T_{\mu \nu} & =0 \\
\nabla_{\mu} F^{\mu \nu} & =0 .
\end{aligned}
$$

where the trace-free Bach tensor $B_{\mu \nu}$ and energymomentum tensor of electromagnetic field $T_{\mu \nu}$ are defined as

$$
\begin{aligned}
& B_{\mu \nu}=\left(\nabla^{\rho} \nabla^{\sigma}+\frac{1}{2} R^{\rho \sigma}\right) C_{\mu \rho \nu \sigma}, \\
& T_{\mu \nu}=F_{\alpha \mu} F_{\nu}^{\alpha}-\frac{1}{4} g_{\mu \nu} F_{\alpha \beta} F^{\alpha \beta} .
\end{aligned}
$$

For a static black hole with spherical symmetry, the metric has the form

$$
\mathrm{d} s^{2}=-h(r) \mathrm{d} t^{2}+\frac{\mathrm{d} r^{2}}{f(r)}+r^{2} \mathrm{~d} \theta^{2}+r^{2} \sin ^{2}(\theta) \mathrm{d} \varphi^{2} .
$$

By substituting eq. (6) into the field equations, we arrive at the differential equations

$$
\begin{aligned}
& r h\left[r f^{\prime} h^{\prime}+2 f\left(r h^{\prime \prime}+2 h^{\prime}\right)\right] \\
& +4 h^{2}\left(r f^{\prime}+f-1+2 \Lambda r^{2}\right)-r^{2} f h^{\prime 2}=0,
\end{aligned}
$$

$$
\begin{aligned}
& f^{\prime \prime}+\frac{r^{2} f h^{\prime 2}+2 r f h h^{\prime}+4\left(f-1+2 \Lambda r^{2}\right) h^{2}}{2 r f h\left(r h^{\prime}-2 h\right)} f^{\prime} \\
& -\frac{3 h f^{\prime 2}}{4 f h-2 r f h^{\prime}}+8 \Lambda \frac{h-f h-r f h^{\prime}-\Lambda r^{2} h}{3 f\left(2 h-r h^{\prime}\right)} \\
& -\frac{r^{3} f h^{\prime}+\left(r^{2} f-r^{2}+\Lambda r^{4}+\kappa Q_{0}^{2}\right) h}{\alpha r^{2} f\left(r h^{\prime}-2 h\right)} \\
& -\frac{r^{3} f h^{\prime 3}-3 r^{2} f h h^{\prime 2}-8(f-1) h^{3}}{2 r^{2} h^{2}\left(r h^{\prime}-2 h\right)}=0, \\
& A_{t}^{\prime}+\sqrt{\frac{h}{f} \frac{Q_{0}}{r^{2}}}=0,
\end{aligned}
$$

where $Q_{0}$ is the electric charge of the black hole. Imposing standard physical boundary conditions implies that $A_{t}(r) \rightarrow 0$ at the cosmological horizon $r_{c}$ (infinity) in an asymptotically (Anti-) de Sitter spacetime. Besides, it is easy to verify that, for uncharged spacetimes, the analytic Schwarzschild (Anti-) de Sitter black-hole solutions satisfy the above field equations. However, this is not true for charged spacetimes. It can be shown straightforwardly that the Reissner-Nordström (Anti-) de Sitter metric does not satisfy the above field equations when $\alpha \neq 0$. The same condition was pointed out in [8] for the case without cosmological constant.

In what follows we apply the shooting method to numerically study the Schwarzschild as well as nonSchwarzschild solutions and their generalizations. We can set $\alpha=\frac{1}{2}$ and $\kappa=1$ without losing generality. After that, we expand the functions $h(r)$ and $f(r)$ around the event horizon $r_{0}$ as follows:

$$
\begin{aligned}
& h(r)=h_{1}\left(r-r_{0}\right)+h_{2}\left(r-r_{0}\right)^{2}+h_{3}\left(r-r_{0}\right)^{3}+\cdots, \\
& f(r)=f_{1}\left(r-r_{0}\right)+f_{2}\left(r-r_{0}\right)^{2}+f_{3}\left(r-r_{0}\right)^{3}+\cdots,
\end{aligned}
$$

where $f_{i}$ and $h_{i}$ are constant coefficient. Since we can always rescale the time coordinate by the transformation $t \rightarrow \frac{t}{\text { const }}$ for any positive constant $C$, then $C \times h(r)$ is always a solution of the field equations if $h(r)$ itself is a solution of the corresponding equations. Therefore, we may choose $f_{1}=h_{1}$ for the sake of simplicity. By substituting eq. (10) into the field equations, all $h_{j}$ and $f_{j}$ with $j \geq 2$ can be expressed in terms of $f_{1}$, for example, $h_{2}$ and $f_{2}$ are given by

$$
\begin{aligned}
h_{2}= & \frac{1-2 f_{1} r_{0}}{r_{0}^{2}}+\frac{r_{0}^{2}-f_{1} r_{0}^{3}-\kappa Q_{0}^{2}}{8 \alpha f_{1} r_{0}^{3}} \\
& -\left(\frac{5}{3}+\frac{1}{3 f_{1} r_{0}}+\frac{3 r_{0}}{8 \alpha f_{1}}\right) \Lambda+\frac{r_{0} \Lambda^{2}}{3 f_{1}}, \\
f_{2}= & \frac{1-2 f_{1} r_{0}}{r_{0}^{2}}-3 \frac{r_{0}^{2}-f_{1} r_{0}^{3}-\kappa Q_{0}^{2}}{8 \alpha f_{1} r_{0}^{3}} \\
& -\left(3-\frac{1}{f_{1} r_{0}}-\frac{3 r_{0}}{8 \alpha f_{1}}\right) \Lambda-\frac{r_{0} \Lambda^{2}}{f_{1}} .
\end{aligned}
$$

Now we are in the position to calculate the numerical black-hole solutions of the field equations.

For the case of asymptotically de Sitter black holes $(\Lambda>0)$ we fix $\Lambda=0.01$ for the sake of convenience. Let 

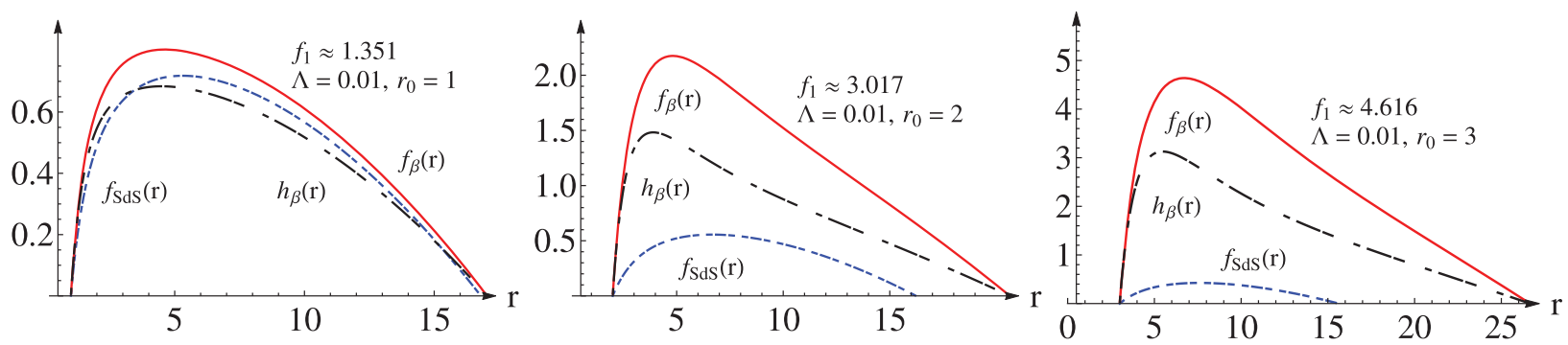

Fig. 1: (Colour online) Schwarzschild de Sitter black hole and non-Schwarzschild de Sitter black hole for $r_{0}=1, r_{0}=2$ and $r_{0}=3$. We have $f(r)=h(r)=f_{S d S}(r)$ for the Schwarzschild de Sitter case, and $f(r)=f_{\beta}(r)$ and $h(r)=h_{\beta}(r)$ for the non-Schwarzschild de Sitter case. Group-I solution (left) and group-II solution (middle, right).
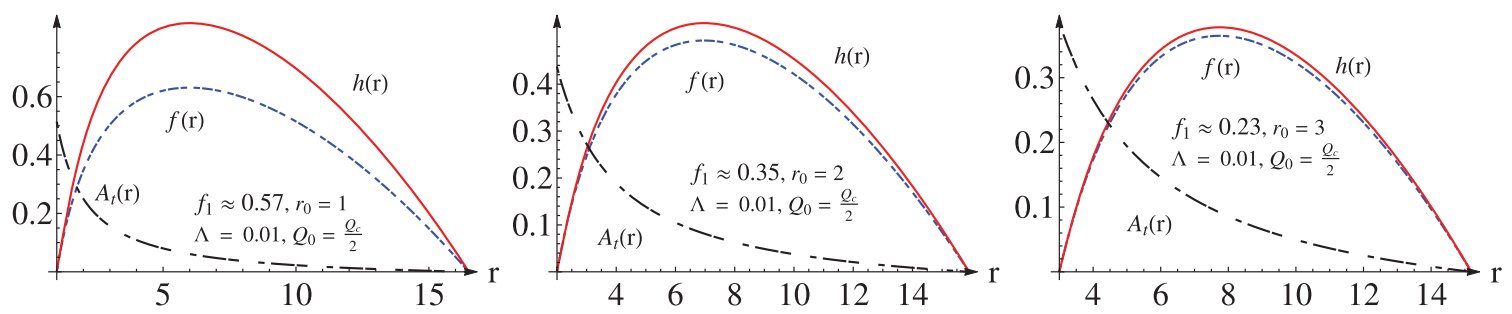

Fig. 2: (Colour online) Numerical charged de Sitter solutions $f(r), h(r)$ and $A_{t}(r)$ belonging to group I with $r_{0}=1,2,3$ and $Q_{0}=\frac{Q_{c}}{2}$.
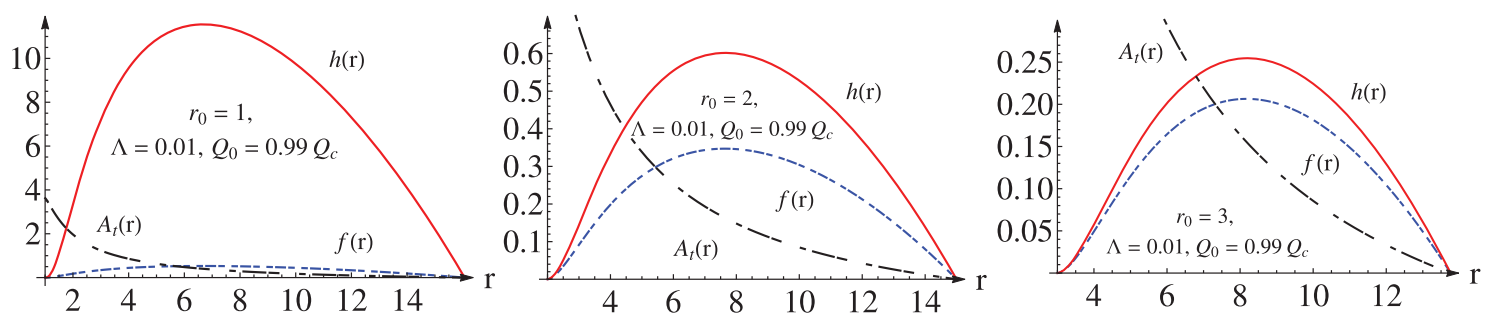

Fig. 3: (Colour online) Numerical charged de Sitter solutions group I (near the extreme case) of $f(r), h(r)$ and $A_{t}(r)$ with $r_{0}=1,2,3$ and $Q_{0}=0.99 Q_{c}$, where $Q_{c}$ is the charge of the extreme de Sitter black hole (see eq. (12)).

us first study the case when $Q_{0}=0$, which corresponds to the uncharged black holes. As mentioned above, the Schwarzschild de Sitter metric is an exact solution of the field equations. The non-Schwarzschild de Sitter solutions were found numerically by the following procedure. Since the de Sitter black hole has an event horizon and a cosmological horizon, $f$ and $h$ should satisfy the boundary conditions to attain zero at both horizons. From the form of eq. (10), it is obvious that the above first boundary condition at the event horizon is automatically satisfied, and the second boundary condition at the cosmological horizon is used as the criterion of the initial value of $f_{1}$ in the shooting method.

At this point we shall introduce a classification to the black-hole solutions that will be used throughout paper. The Schwarzschild (Anti-) de Sitter solutions and those that can be reduced to the Schwarzschild (Anti-) de Sitter solutions under specific conditions are referred to as group-I solution. Similarly, the non-Schwarzschild (Anti-) de Sitter solutions as well as those can be reduced to the
non-Schwarzschild (Anti-) de Sitter solutions under specific conditions are referred to as group II.

The (non-)charged asymptotically de Sitter black-hole solutions found are shown in figs. 1, 2, 3, 4 for some given values of $r_{0}$ and $Q_{0}$.

When $Q_{0}=0$ and $\alpha \neq 0$, we have two groups of solutions, such that group-I solutions reduce to Schwarzschild de Sitter black hole and group-II solutions reduce to nonSchwarzschild de Sitter black hole, respectively. In fig. 1, one finds that the difference between Schwarzschild de Sitter black hole and non-Schwarzschild de Sitter black hole increases as $r_{0}$ increases. In particular, the cosmological horizons are larger for non-Schwarzschild de Sitter black hole than for Schwarzschild de Sitter black hole. Besides, it is noted that $h \neq f$ for non-Schwarzschild de Sitter black holes.

When $Q_{0} \neq 0$ and $\alpha \neq 0$, we can also separate the solutions into two groups, which reduce to Schwarzschild de Sitter black hole and non-Schwarzschild de Sitter black hole, respectively as $Q_{0} \rightarrow 0$. In fig. 2 some charged 

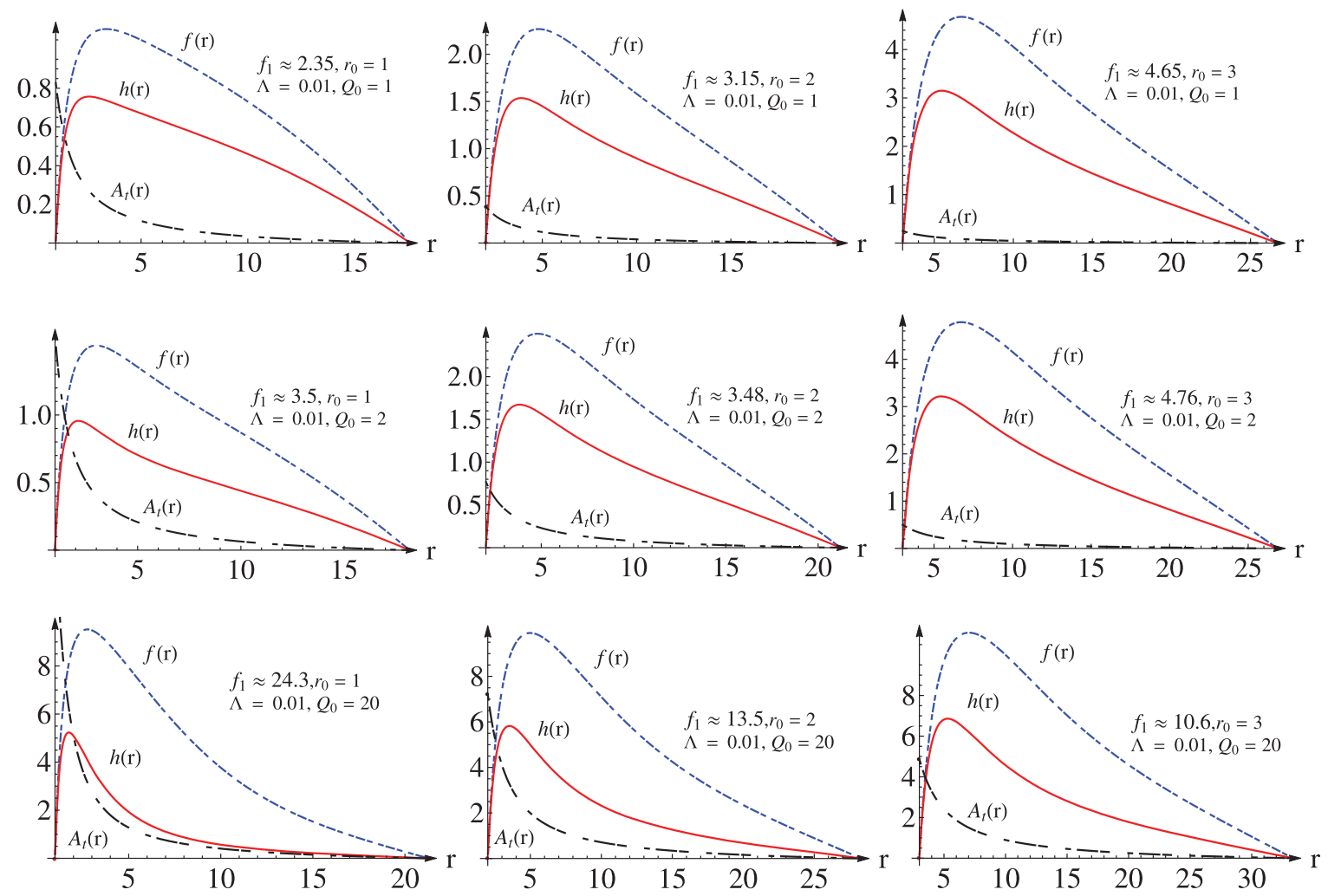

Fig. 4: (Colour online) Charged de Sitter solutions belonging to group II. Displayed are $f(r), h(r)$ and $A_{t}(r)$ with $r_{0}=1,2,3$ and $Q_{0}=1,2,20$.
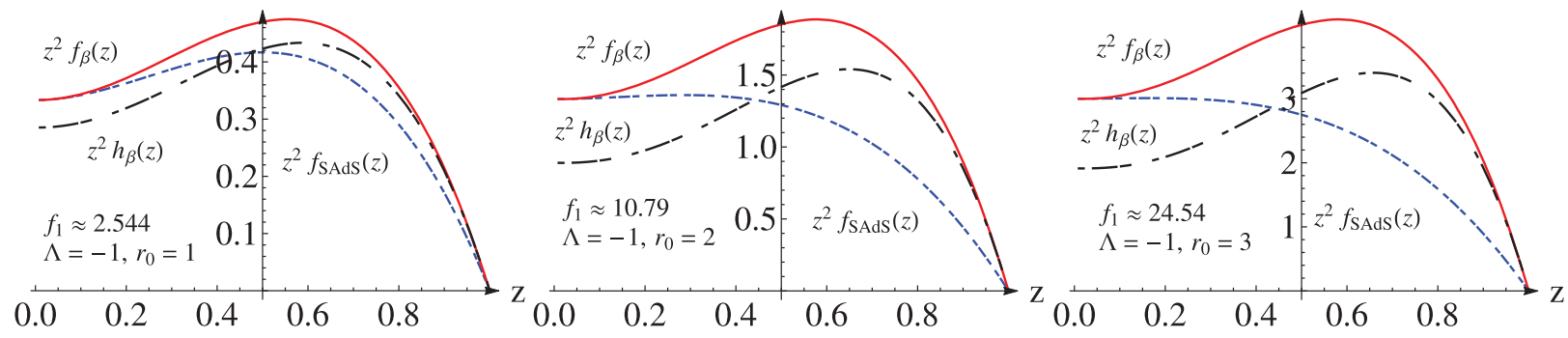

Fig. 5: (Colour online) Schwarzschild and non-Schwarzschild Anti-de Sitter black holes for $r_{0}=1, r_{0}=2$ and $r_{0}=3$. We have $f(r)=h(r)=f_{A S d S}(r)$ for the Schwarzschild Anti-de Sitter case, and $f(r)=f_{\beta}(r)$ and $h(r)=h_{\beta}(r)$ for the non-Schwarzschild Anti-de Sitter case.

asymptotic de Sitter solutions are presented for different values of $r_{0}$ and $Q_{0}=\frac{Q_{c}}{2}$.

For group I we found that there is a maximal physical value of the charge which is attained when the black hole becomes an extreme de Sitter black hole. This critical value is given by

$$
Q_{c}^{d S}=\frac{r_{0}}{\sqrt{3 \kappa}} \sqrt{\left(r_{0}^{2} \Lambda-1\right)(8 \alpha \Lambda-3)},
$$

and the extreme solution is reached imposing, additionally, $f_{1}=0$. In fig. 3 we show some examples of the above results. It is noteworthy that it is very difficult to directly calculate numerically the extreme case, so that we only study the case where the charge $Q_{0}$ is very close to the extreme value $Q_{c}$. In the plots, we choose $Q_{0}=0.99 Q_{c}$. We note that the difference between $f(r)$ and $h(r)$ decreases with decreasing $r_{0}$. In fig. 4 we have the groupII solutions. It turns out that group II does not have an upper limit of the charge differently from group I. This interesting propriety was already pointed out in [8] for the case without cosmological constant. For both groups of solutions, although they can be reduced to the (non-)Schwarzschild de Sitter black hole at zero charge limit, none of them has Reissner-Nordström de Sitter metric as valid solution. It can be related with a non-trivial coupling between the electric charge and the geometry through Weyl scalar and that appears in eq. (11).

For the case of asymptotically de Anti-de Sitter black holes $(\Lambda<0)$ there is not cosmological horizon. Thus, 

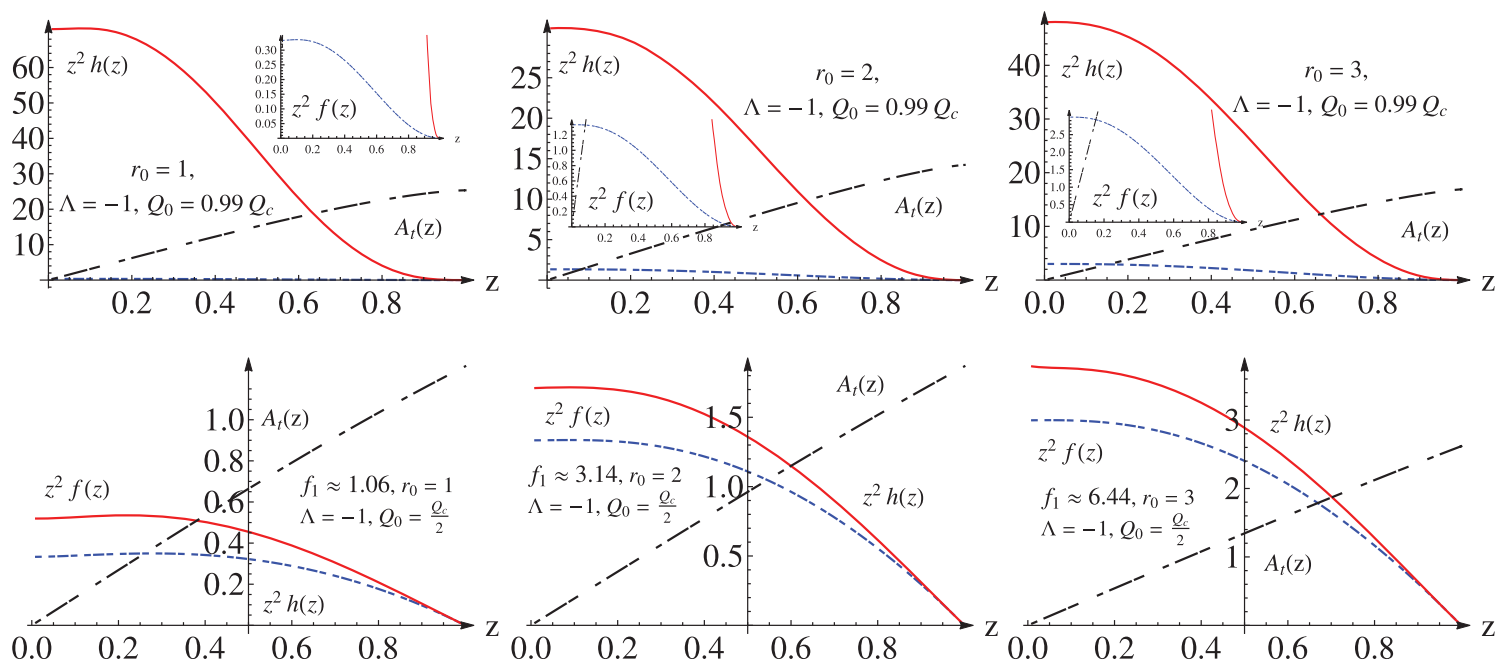

Fig. 6: (Colour online) Anti-de Sitter solutions belonging to group I. We display $f(r), h(r)$ and $A_{t}(r)$ with $r_{0}=1,2,3$ and $Q_{0}=0.99 Q_{c}, \frac{Q_{c}}{2}$, where $Q_{c}$ is the charge of the extreme Anti-de Sitter black hole.
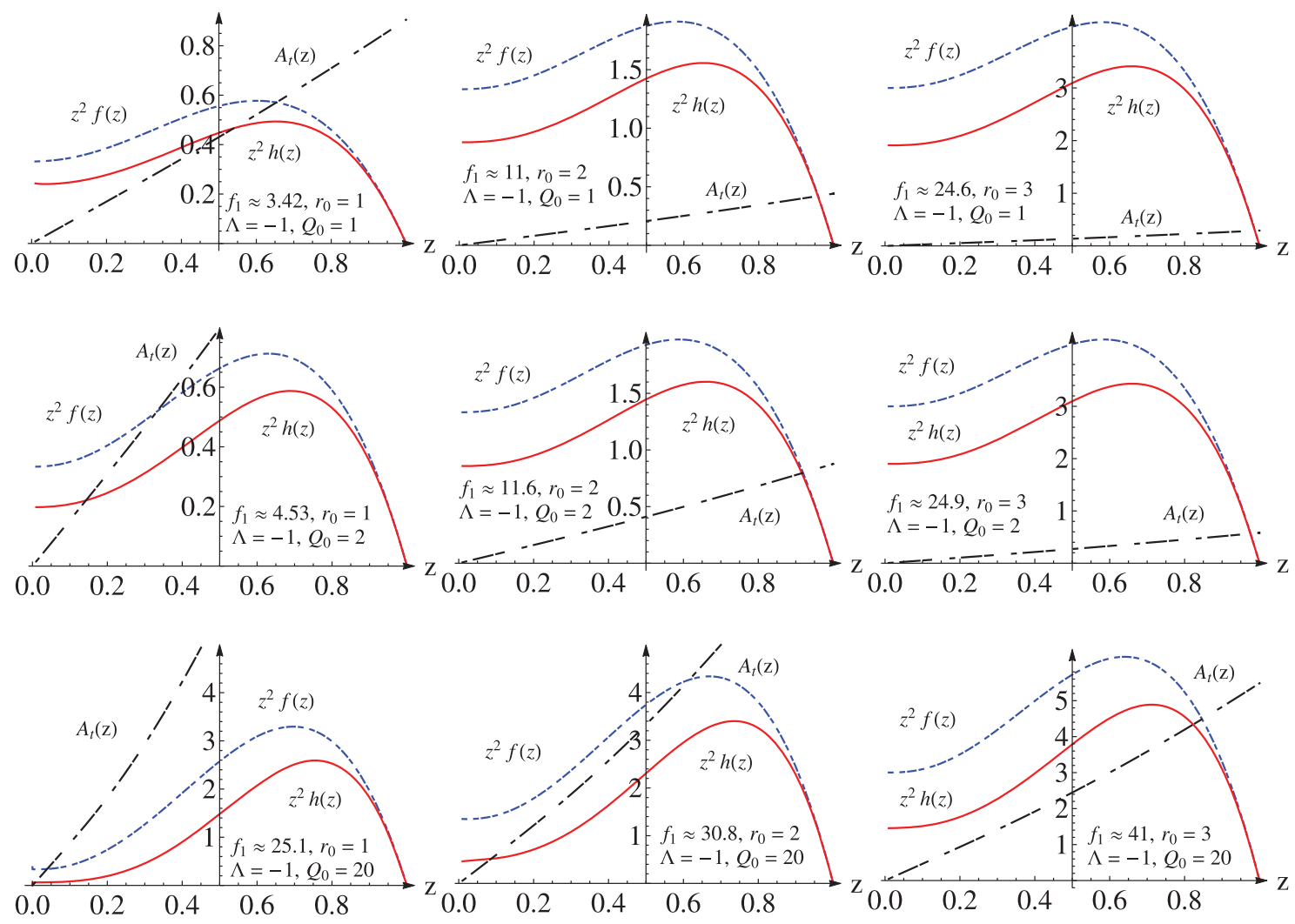

Fig. 7: (Colour online) Numerical Anti-de Sitter solutions group II of $f(r), h(r)$ and $A_{t}(r)$ with $r_{0}=1,2,3$ and $Q_{0}=1,2,20$.

the conditions used previously that $f$ and $h$ vanish at the cosmological horizon is no more valid and this implies that one has to adopt the boundary condition at infinity as the criterion of the shooting method.

Since it is known that $f$ and $h$ approach asymptotically the form $r^{2}$ as $r \rightarrow \infty$, we introduce the coordinate transformation $z=\frac{r_{0}}{r}$, so that the function of $f$ and $h$ become $f=f(z)$ and $h=h(z)$ where $0 \leq z \leq 1$. When $z=1$, the corresponding radial coordinate is the event horizon $r_{0}$, and $z=0$ corresponds to $r \rightarrow \infty$. Therefore, with this transformation it is possible to apply the boundary conditions at finite values of $z=0$ and $z=1$. In our calculations, the condition that $f$ and $h$ are proportional to $z^{-2}$ at $z=0$ is used as the criterion for the shooting method: at $z=0$, the functions $z^{2} f(z)$ and $z^{2} h(z)$ should be positive and their derivatives with respect to $z$ should 
vanish. We show the resulting Anti-de Sitter black hole in figs. $5,6,7$.

Without loss of generality, we set $\Lambda=-1$. Similar to case of de Sitter spacetime, we find that the field equations possess two groups of solutions, which reduce to Schwarzschild Anti-de Sitter and non-Schwarzschild Antide Sitter black holes, respectively as $Q_{0} \rightarrow 0$. We show the uncharged Anti-de Sitter solutions in fig. 5. It is observed that the difference between Schwarzschild Anti-de Sitter and non-Schwarzschild Anti-de Sitter black holes increases as $r_{0}$ increases.

Again, the Reissner-Nordström Anti-de Sitter metric is not a solution of the field equations, while the group-I solutions reduce to Schwarzschild Anti-de Sitter black hole as $Q_{0} \rightarrow 0$. Surprisingly, for this theory of gravity, an "extreme" Anti-de Sitter black-hole solution is possible.

The critical charge $Q_{c}^{A d S}$ of the "extreme" Anti-de Sitter black hole for group I is determined by

$$
Q_{c}^{A d S}=\frac{r_{0}}{\sqrt{3 \kappa}} \sqrt{\left(r_{0}^{2}|\Lambda|+1\right)(8 \alpha|\Lambda|+3)} .
$$

However, for the group-II solutions, no maximal charge is found. We show numerical solutions for groups I and II in fig. 6 and fig. 7, respectively.

In this letter, we investigated (Anti-) de Sitter electrically charged static black-hole solutions of Einstein-Weyl gravity. Two groups of solutions were found for the theory. Group I is characterized by an upper limit for the black hole's charge corresponding to the extreme (Anti-) de Sitter black hole, while group II does not possess any maximal charge. These conclusions are consistent with the results found in $[6,8]$. Recently, the quasinormal modes of non-Schwarzschild solutions have been studied in [11] and it was shown that the black-hole spacetime is stable. It is therefore interesting to investigate the quasinormal modes and stability of the charged static solutions of Einstein-Weyl theory in (Anti-) de Sitter spacetime. In particular the latter may have important implications on the AdS/CFT correspondence since the non-Schwarzschild AdS black hole could be a new thermal state in CFT.

This work is supported in part by the Brazilian funding agencies FAPESP, FAPEMIG, CNPq, CAPES, and by the Chinese funding agencies NNSFC under contract Nos. 11573022 and 11375279.

\section{REFERENCES}

[1] 't Hooft G. and Veltman M. J. G., Ann. Henri Poincaré Phys. Theor. A, 20 (1974) 69.

[2] Stelle K. S., Phys. Rev. D, 16 (1977) 953.

[3] Lin K., de Oliveira J. and Abdalla E., Phys. Rev. D, 90 (2014) 124071.

[4] Zhu Z., Zhang S. J., Pellicer C. E., Wang B. and Abdalla E., Phys. Rev. D, 90 (2014) 044042.

[5] Konoplya R. A. and Zhidenko A., Phys. Rev. Lett., 103 (2009) 161101; Cardoso V., Lemos M. and Marques M., Phys. Rev. D, 80 (2009) 127502.

[6] Lü H., Perkins A., Pope C. N. and Stelle K. S., Phys. Rev. Lett., 114 (2015) 171601 (arXiv:1502.01028 [hep-th]).

[7] Lü H., Perkins A., Pope C. N. and Stelle K. S., Phys. Rev. D, 92 (2015) 124019 (arXiv:1508.00010 [hep-th]).

[8] Lin K., Pavan A. B., Flores-Hidalgo G. and Abdalla E., arXiv:1605.04562 [gr-qc].

[9] Aharony O., Gubser S. S., Maldacena J. M., Oogury H. and Oz Y., Phys. Rep., 323 (2000) 183.

[10] Nelson W., Phys. Rev. D, 82 (2010) 104026.

[11] Cai Y-F., Gheng G., Liu J., Wang M. and Zhang H., JHEP, 01 (2016) 108 (arXiv:1508.04776 [hep-th]). 